\section{Prevalência de fatores de risco para doenças cardiovasculares entre homens de uma população urbana do Sudeste do Brasil}

\author{
Prevalence of risk factors for cardiovascular \\ diseases in an urban male population in \\ Southeast Brazil
}

${ }^{1}$ Mestrado Profissional em Saúde da Família, Universidade Estácio de Sá, Rio de Janeiro, Brasil. 2 Instituto de Medicina Social, Universidade do Estado do Rio de Janeiro, Rio de Janeiro, Brasil.

Correspondência E. B. B. D. Van Eyken Mestrado Profissional em Saúde da Família, Universidade Estácio de Sá. Rua do Riachuelo 27, 60 andar, Rio de Janeiro, $R J$ 20230-010, Brasil. elisa.vaneyken@gmail.com

\begin{abstract}
Surveillance of cardiovascular risk factors has been recommended worldwide. The current study aimed to estimate the prevalence of smoking, overweight/obesity, insufficient physical activity, arterial hypertension, and pre-hypertension among men covered by the Family Health Program in urban Juiz de Fora, Minas Gerais State, in Southeast Brazil. A household survey was performed with a systematic sample of 217 men 20 to 49 years of age, from a list of residents within this age range. Prevalence of risk factors was high, with overweight/obesity the most frequent (43.3\%; 95\%CI: 36.6-50.2). Men classified as sedentary or with irregular physical activity totaled 28\% (95\%CI: 22.2-34.6). Smoking was reported by 25.3\% (95\% CI: 19.7-31.7). Overt hypertension was present in 24\% (95\%CI: 18.4-30.2) and borderline hypertension in 19.4\% (95\%CI: 14.3-25.2). Two or more simultaneous risk factors were found in $45.2 \%$ of the sample. The high prevalence rates suggest that health promotion strategies need to be intensified in the male population.
\end{abstract}

Cardiovascular Diseases; Men's Health; Risk Factors
Elisa Beatriz Braga Dell'Orto Van Eyken 1 Claudia Leite Moraes 1,2

\section{Introdução}

Segundo a Organização Mundial da Saúde (OMS), as doenças cardiovasculares são responsáveis por $30 \%$ do total de mortes no mundo. A projeção da OMS é que esse grupo de doenças seja a primeira causa de morte em todos os países em desenvolvimento até 20101 .

Atualmente, sugere-se que mais de $80 \%$ dos casos de morte por doenças cardiovasculares estejam associados a fatores de risco já conhecidos 2 . São considerados mais importantes os fatores que apresentam alta prevalência em muitas populações; os que têm impacto independente e significante no risco para doenças isquêmicas e acidente vascular cerebral; e os modificáveis ou passíveis de controle. Por apresentarem esses três critérios de relevância, vem sendo enfatizado o controle do diabetes mellitus, da obesidade, da inatividade física, do uso do tabaco, da hiperlipidemia e da hipertensão arterial 2.

Dentre os fatores de risco modificáveis, a hipertensão arterial é considerada o mais importante para as doenças isquêmicas e para o acidente vascular cerebral 2 . Nos últimos anos, alguns autores têm chamado a atenção para a necessidade de rediscussão dos níveis pressóricos considerados ideais, pois parece haver uma relação linear entre o risco de morte por causa vascular e os valores da pressão arterial, mesmo dentre o espectro de valores considerados normais $3,4,5$. 
Outro fator de risco modificável importante é o tabagismo. Estima-se que esse hábito seja a principal causa de morte evitável no mundo em função de sua atuação como precursor de diversas patologias e sua alta prevalência. A magnitude do problema é identificada ao se considerar a estimativa da OMS de que cerca de $1 / 3$ da população mundial adulta seja fumante 6 . Assim como o hábito de fumar, a inatividade física, a obesidade e o sobrepeso também vêm sendo ressaltados pela instituição como um importante entrave para o controle das doenças cardiovasculares 7 . Estima-se que o sedentarismo, ainda que de forma dependente de outros fatores, seja responsável por $22 \%$ das doenças isquêmicas do coração 8 e que a obesidade e o sobrepeso já atinjam 1 bilhão de pessoas no mundo 7 .

No Brasil, estudos de base populacional e em populações específicas confirmaram a alta prevalência desses fatores 9,10,11,12,13,14,15,16,17,18. Dentre esses se destaca o Inquérito Domiciliar sobre Comportamentos de Risco e Morbidade Referida de Doenças e Agravos não Transmissíveis: Brasil, 15 Capitais e Distrito Federal, 200220038 , cujos dados revelam a alta prevalência do tabagismo $(12,9 \%$ a $25,2 \%)$, da inatividade física $(28,2 \%$ a $54,5 \%)$, da hipertensão arterial $(7,4 \%$ a $59 \%)$, do sobrepeso $(23 \%$ a $33,5 \%)$ e da obesidade $(8,1 \%$ a $12,9 \%)$ nas diferentes faixas etárias e regiões do país.

Devido à relevância das doenças cardiovasculares no perfil de morbimortalidade mundial, em 2002 a OMS reafirmou como estratégia fundamental para a redução do problema o monitoramento e vigilância das doenças cardiovasculares e de seus principais fatores de risco 7. A vigilância epidemiológica para doenças e agravos não-transmissíveis e dos seus fatores de risco já é uma realidade em diversos países. Nos Estados Unidos, por exemplo, o mais conhecido e amplo acompanhamento de fatores de risco para doenças e agravos não-transmissíveis é o Behavioral Risk Factor Surveillance Survey System (BRFSS), inquérito populacional que monitora todos os estados americanos por meio de entrevista mensal conduzida por telefone ${ }^{19}$. Outra iniciativa nesse sentido foi o The WHO Mega Country Health Promotion Network, criado com a intenção de formar uma rede de cooperação entre os 11 países com 100 milhões ou mais de habitantes, que juntos constituem mais de $60 \%$ da população mundial 20. Com essa rede seria possível melhorar as evidências para as ações de saúde usando-se coleta de dados por meio de um instrumento e protocolo com questões-padrão centrais 20 .

Apesar do avanço na produção nacional de conhecimento, alguns autores têm apontado para a insuficiência de pesquisas que permitam um acompanhamento sistemático da prevalência desses fatores de risco na população ${ }^{21,22}$. A carência de um sistema de vigilância nacional para as doenças e agravos não-transmissíveis e seus fatores de risco gera uma desvantagem para o Brasil em relação aos países desenvolvidos 13,23. Para minorar essa desvantagem, o Ministério da Saúde desencadeou em 2000 a estruturação e operacionalização de um sistema de vigilância que tem como objetivo relacionar os sistemas de informação já existentes com os resultados de inquéritos populacionais de abrangência nacional a serem realizados a cada cinco anos, inquéritos municipais e estudos de populações mais vulneráveis 24 .

Integrante da atenção básica à saúde, a Saúde da Família é uma estratégia de mudança do Sistema Único de Saúde (SUS) de um modelo meramente assistencial e hospitalocêntrico, para um baseado na atenção básica como sua porta de entrada. Mais abrangente do que o modelo tradicional, a Saúde da Família engloba ações de promoção da saúde, prevenção de doenças, assistência aos agravos mais prevalentes, recuperação e reabilitação. Por essas características, o Programa Saúde da Família (PSF) tem um grande potencial de se integrar à rede de vigilância em saúde voltada para as doenças crônicas. A cobertura da estratégia, que hoje já atinge 50 milhões de brasileiros, reforça a necessidade do debate sobre as possibilidades, limites e desafios de sua participação nas propostas de prevenção desses agravos 25 .

Visando a ressaltar o potencial do PSF na implementação de ações de vigilância das doenças cardiovasculares e ampliar o conhecimento sobre o tema em cidades de médio porte do Brasil, este estudo teve como objetivo estimar a prevalência e o grau de simultaneidade do tabagismo, da inatividade física, do sobrepeso/obesidade, da pressão arterial limítrofe e da hipertensão arterial em uma população masculina adulta residente na cidade de Juiz de Fora, Estado de Minas Gerais. A escolha desse subgrupo populacional se deveu ao seu maior coeficiente de mortalidade por doenças cardiovasculares, cerca de $50 \%$ maior do que na população feminina em adultos jovens 26 , e a distância entre esses homens e os serviços de saúde, o que dificulta o monitoramento dos fatores de risco e o cuidado à saúde 27. Como objetivo secundário, pretendeu observar a distribuição dos diferentes fatores em relação a características sócio-demográficas dessa população.

Além de produzir informações que orientem as políticas de promoção da saúde e redução das doenças cardiovasculares nos níveis local 
e nacional, o presente estudo também pretende contribuir para uma maior incorporação da vigilância à saúde nas atividades da atenção básica, especialmente na Estratégia Saúde da Família.

\section{Métodos}

\section{Desenho de estudo}

Trata-se de um estudo transversal de base populacional, desenvolvido na Estratégia Saúde da Família da unidade básica de saúde do Alto Grajaú, localizada na cidade de Juiz de Fora.

\section{Cenário da pesquisa}

A cidade de Juiz de Fora, localizada na região da zona da mata, em Minas Gerais, possui uma área territorial de $1.437 \mathrm{~km}^{2}$ e é a cidade pólo da saúde de 38 municípios (Instituto Brasileiro de Geografia e Estatística. Cidades@.http:// www.ibge.gov.br/cidadesat/, acessado em 30/ Mar/2006. Prefeitura de Juiz de Fora. História. http://www.juizdefora.mg.gov.br/, acessado em 29/Mar/2006). Segundo o Censo Demográfico 2000 (http://ww.ibge.gov.br), Juiz de Fora possuía 456.796 habitantes, sendo 99,17\% residentes na área urbana da cidade.

A unidade básica de saúde do Alto Grajaú funciona com duas equipes de Saúde da Família que atendem as áreas de números 17 e 63 do município. Cada área de abrangência das equipes é subdivida em seis microáreas. A população total das duas áreas, em 2006, era de 2.245 famílias que compreendiam 7.680 pessoas (Departamento de Informática do SUS. Indicadores e dados básicos - Brasil - 2005. IDB-2005. http:// tabnet.datasus.gov.br/cgi/idb2005/matriz.htm, acessado em 02/Dez/2006), correspondendo a $1,5 \%$ do total de famílias cobertas pela Estratégia Saúde da Família de Juiz de Fora que, por sua vez, apresenta atualmente $50 \%$ de cobertura via-a-vis o conjunto de moradores da cidade.

\section{População alvo e população fonte}

A população alvo da pesquisa foi constituída por homens, com idades entre 20 e 49 anos, residentes em áreas cobertas pela Estratégia Saúde da Família de Juiz de Fora. A população fonte foi composta por todos os 692 homens com idades de 20 a 49 anos, da área 17, excluindo-se a microárea 5, da referida unidade básica de saúde, cadastrados até janeiro de 2006.
Tamanho e estratégia de seleção da amostra do estudo

A amostra foi constituída com base em uma malha amostral formada pelo conjunto de homens dessa faixa etária residentes na área de abrangência da referida unidade, por meio de uma estratégia sistemática de seleção.

O tamanho amostral foi definido baseandose na fórmula: $\mathrm{n}=\mathrm{Z}^{2} \mathrm{PQ} / \mathrm{d}^{2}$, sendo $\mathrm{n}=$ tamanho amostral mínimo; $\mathrm{Z}$ = variável reduzida; $\mathrm{P}=$ probabilidade de encontrar o fenômeno estudado. A maior estimativa de prevalência dos fatores de risco de interesse, na faixa etária acima de 25 anos, na população de homens na cidade de Belo Horizonte, Estado de Minas Gerais, pelo Inquérito Domiciliar sobre Comportamento de Risco e Morbidade Referida de Doenças e Agravos Não Transmissíveis: Brasil, 15 Capitais e Distrito Federal, 2002-2003 8 para população insuficientemente ativa levou à adoção de um valor igual a $42,5 \%$ para a probabilidade dos eventos serem encontrados: $\mathrm{Q}=1-\mathrm{P} ; \mathrm{d}=$ precisão desejada, cujo valor foi de $5 \%$.

O tamanho amostral mínimo previsto foi de 244 pessoas. Considerando a possibilidade de perdas, acrescentou-se $10 \%$ a essa estimativa.

\section{- Coleta de dados}

O trabalho de campo transcorreu durante os meses de junho a outubro de 2006. Visando a aumentar a aceitabilidade da pesquisa pela população cadastrada, antes da ida aos domicílios, os agentes comunitários de saúde entregavam uma carta-convite aos usuários para a participação na entrevista. Para reduzir possíveis riscos para a equipe de entrevistadores, os agentes os acompanhavam às visitas em áreas de difícil acesso. Os dados foram colhidos mediante aplicação de questionário estruturado, em entrevistas domiciliares face a face, por uma equipe de 12 alunos de uma faculdade de fisioterapia da cidade. Os entrevistadores foram treinados para a aferição da pressão arterial de acordo com as IV Diretrizes Brasileiras de Hipertensão Arterial 28 e para a aplicação do questionário. Houve um estudo piloto de 20 a 27 de abril de 2006, na instituição de ensino à qual estavam vinculados, quando foram aferidas as pressões de 357 pessoas e aplicados 36 questionários.

\section{- Instrumentos da pesquisa}

O instrumento de coleta de dados foi composto por módulos de questionários já testados e validados em estudos anteriores realizados no Brasil 8,29,30,31,32. O primeiro módulo compreendia 
características demográficas básicas e informações sobre indicadores sócio-econômicos. Para a captação das variáveis demográficas utilizou-se o questionário da Pesquisa Nacional por Amostra de Domicílios (PNAD) ${ }^{31}$. Para apreensão da situação econômica do respondente foi utilizado o Critério de Classificação Econômica Brasil 30. Esse critério apresenta uma classificação em sete níveis a partir do total da soma da posse dos bens e utensílios domésticos e da escolaridade da pessoa de maior renda no domicílio, sendo estas as classes: A1, A2 (classe alta), B1, B2, (classe média) C (média baixa), D (pobre) e E (muito pobre). Na análise de dados do presente estudo utilizou-se a variável de forma agrupada em três níveis, a saber: A e B; C; D e E.

O segundo módulo foi composto por perguntas sobre saúde relacionadas à pressão arterial, ao peso e à altura corporais. Foram utilizadas questões do módulo de pressão arterial Questionário Individual Tipo A do inquérito domiciliar sobre comportamento de risco e morbidade referida de doenças e agravos não-transmissíveis ${ }^{8}$. Para a caracterização da situação nutricional dos respondentes utilizou-se o índice de massa corporal (IMC) por meio da informação do peso e da estatura referidos 29 . A classificação dos indivíduos de acordo com o IMC se baseou na proposta preconizada pela OMS 7 .

O terceiro módulo constou de dados referentes à atividade física. O instrumento de coleta foi a versão 8 do International Physical Activity Questionary (IPAQ) ${ }^{32}$, aplicado por entrevistador, levando em consideração a semana anterior à entrevista. A classificação inicial como "muito ativos", "ativos", "irregularmente ativos" e "sedentários" seguiu o consenso entre o Centro de Estudos do Laboratório de Aptidão Física de São Caetano do Sul e o Centers for Disease Control and Prevention 15 , tendo sido considerados como dois grupos, "muito ativos/ativos" e "irregularmente ativos/sedentários”, para a análise da distribuição da atividade física em relação às características sócio-demográficas da população do estudo.

O quarto módulo abordou questões relacionadas ao tabagismo, também aproveitadas do inquérito realizado pelo Ministério da Saúde entre 2002 e $2003{ }^{8}$. A pressão arterial sistêmica foi aferida e classificada segundo as normas das $I V$ Diretrizes Brasileiras de Hipertensão Arterial 28, com aparelho de pressão aneróide da marca BD (BD-Brasil, Curitiba), tamanho adulto médio (24$30 \mathrm{~cm}$ ), periodicamente calibrado.

\section{- Processamento e análise dos dados}

Para a entrada de dados e o controle de qualidade foi utilizado o programa Epi Info 3.3.2 (Centers for
Disease Control and Prevention, Atlanta, Estados Unidos). O programa SPSS (SPSS Inc., Chicago, Estados Unidos) foi usado para o processamento e análise de dados. Estimou-se a prevalência dos fatores de risco e seus respectivos intervalos de 95\% de confiança (IC95\%) na população total entrevistada e nos vários subgrupos criados com base nas características sócio-demográficas estudadas. Para avaliação da heterogeneidade das proporções nos subgrupos utilizou-se o teste qui-quadrado. Assumiu-se uma distribuição binomial para a estimação dos intervalos de confiança.

A pesquisa foi autorizada pela Secretaria de Saúde do município onde foi desenvolvida, e aprovada pelo Comitê de Ética da universidade brasileira envolvida no projeto, uma vez que respeitou os princípios éticos contidos na Declaração de Helsinki e na Resolução $n^{o}$. 196/96 do Conselho Nacional de Saúde.

\section{Resultados}

Em relação à situação sócio-econômica e demográfica, os respondentes se distribuíram entre as faixas etárias de 20 a 29 anos (33,2\%), 30 a 39 $(28,1 \%)$ e 40 a 49 anos (38,7\%). Identificaram-se como brancos $51,6 \%$ e como negros ou mulatos $47,4 \%$. Referiram ser casados ou estar em alguma união estável 48,8\% dos entrevistados. Mais de $60 \%$ dos pesquisados se identificaram como a pessoa que mais contribui com o orçamento domiciliar, sendo considerados como o chefe da família. Cerca de $40 \%$ dos respondentes completaram o Ensino Médio, porém, cerca de 30\% não haviam completado o Ensino Fundamental. De acordo com o Critério de Classificação Econômica, a amostra estudada se distribuiu prioritariamente entre as classes B, C e D, estando $51,2 \%$ dos entrevistados concentrados na classe C. Em torno de $70 \%$ dos homens pesquisados eram naturais da própria cidade do estudo e $81,1 \%$ relataram alguma atividade remunerada.

Mais de $80 \%$ dos homens entrevistados apresentavam pelo menos um dos fatores de risco estudados. Na análise da simultaneidade dos mesmos, observou-se que $45,2 \%$ da população apresentavam dois ou mais fatores de risco, sendo que $32,7 \%$ apresentaram dois fatores concomitantes, $12 \%$ acumularam três dos fatores e em $0,5 \%$ dos entrevistados os quatro fatores de risco do estudo foram encontrados. A prevalência de apenas um dos fatores de risco estudados foi de $37 \%$.

Como pode ser observado na Tabela 1, o sobrepeso/obesidade apresentou a maior prevalência entre os fatores estudados (43,3\%). Em 
ordem decrescente de magnitude seguiram-se o sedentarismo/atividade física irregular; o tabagismo; a hipertensão arterial; e a pressão arterial limítrofe.

A Tabela 2 apresenta a população de estudo em relação à situação nutricional. Dos 209 entrevistados que referiram o peso e a altura, cinco foram classificados como subnutridos, tendo sido analisados agrupados com o IMC normal. A obesidade/sobrepeso foi mais prevalente nos maiores de trinta anos e entre os casados. Apesar de mais freqüente entre os extremos das classes econômicas, entre os brancos e os de menor escolaridade, as diferenças não foram estatisticamente significativas.

As características da população estudada com relação à prática da atividade física podem ser observadas na Tabela 3. As com maior asso-
Tabela 1

Prevalência de tabagismo, sedentarismo/atividade física irregular, sobrepeso/obesidade, hipertensão e pressão arterial limítrofe, em homens entre 20 e 49 anos adscritos à Estratégia Saúde da Família da unidade básica de saúde do Alto Grajaú, Juiz de Fora, Minas Gerais, Brasil, em 2006

\begin{tabular}{llll}
\hline Fatores de risco & $\mathbf{n}$ & $\%$ & IC95\% \\
\hline Sobrepeso/Obesidade & 94 & 43,3 & $36,6-50,2$ \\
Atividade física irregular/Sedentarismo & 61 & 28,0 & $22,2-34,6$ \\
Tabagismo & 55 & 25,3 & $19,7-31,7$ \\
Hipertensão arterial & 52 & 24,0 & $18,4-30,2$ \\
Pressão arterial limítrofe & 42 & 19,4 & $14,3-25,2$ \\
\hline
\end{tabular}

Tabela 2

Prevalência de índice de massa corporal (IMC) em homens entre 20 e 49 anos, adscritos à Estratégia Saúde da Família da unidade básica de saúde do Alto Grajaú, Juiz de Fora, Minas Gerais, Brasil, em 2006.

\begin{tabular}{|c|c|c|c|}
\hline \multirow[t]{2}{*}{ Características sócio-demográficas } & \multirow[t]{2}{*}{$\mathbf{n}$} & \multicolumn{2}{|c|}{ Sobrepeso/Obesidade } \\
\hline & & $\%$ & IC95\% \\
\hline \multicolumn{4}{|l|}{ Faixa etária (anos) } \\
\hline$<30$ & 68 & 27,9 & $17,7-40,1$ \\
\hline$\geq 30$ & 141 & 53,2 & $44,6-61,6$ \\
\hline Valor de p & 0,001 & & \\
\hline \multicolumn{4}{|l|}{ Cor/Raça } \\
\hline Branca & 110 & 50,0 & $40,3-59,7$ \\
\hline Negra e mulatos & 97 & 39,2 & $29,4-49,6$ \\
\hline Valor de $p$ & 0,118 & & \\
\hline \multicolumn{4}{|l|}{ Estado civil } \\
\hline Casado & 104 & 55,8 & $45,7-65,5$ \\
\hline Solteiro e outros & 105 & 34,3 & $25,3-44,3$ \\
\hline Valor de p & 0,002 & & \\
\hline \multicolumn{4}{|l|}{ Escolaridade } \\
\hline Até o Ensino Médio incompleto & 104 & 48,1 & $38,2-58,1$ \\
\hline Ensino Médio completo ou mais & 105 & 41,9 & $32,2-51,9$ \\
\hline Valor de $\mathrm{p}$ & 0,370 & & \\
\hline \multicolumn{4}{|l|}{ Classificação econômica * } \\
\hline Classes A e B & 87 & 50,6 & $39,6-61,5$ \\
\hline Classe C & 106 & 39,6 & $30,3-49,6$ \\
\hline Classes D e E & 16 & 50,0 & $24,7-75,3$ \\
\hline Valor de $\mathrm{p}$ & 0,288 & & \\
\hline \multicolumn{4}{|l|}{ Situação de trabalho } \\
\hline Com trabalho & 172 & 47,7 & $40,0-55,4$ \\
\hline Sem trabalho & 37 & 32,4 & $18,0-49,8$ \\
\hline Valor de $\mathrm{p}$ & 0,091 & & \\
\hline \multicolumn{4}{|l|}{ Local de nascimento } \\
\hline Juiz de Fora & 143 & 43,4 & $35,1-51,9$ \\
\hline Outros & 66 & 48,5 & $36,0-61,1$ \\
\hline Valor de $p$ & 0,488 & & \\
\hline
\end{tabular}

* De acordo com o critério da Associação Brasileira de Empresas de Pesquisa 30. 
Prevalência de atividade física em homens entre 20 e 49 anos, adscritos à Estratégia Saúde da Família da unidade básica de saúde do Alto Grajaú, Juiz de Fora, Minas Gerais, Brasil, em 2006.

\begin{tabular}{|c|c|c|c|}
\hline \multirow[t]{2}{*}{ Características sócio-demográficas } & \multirow[t]{2}{*}{$\mathbf{n}$} & \multicolumn{2}{|c|}{ Sedentário/Irregularmente ativo } \\
\hline & & $\%$ & IC95\% \\
\hline \multicolumn{4}{|l|}{ Faixa etária (anos) } \\
\hline$<30$ & 72 & 15,2 & $7,9-25,7$ \\
\hline$\geq 30$ & 145 & 34,5 & $26,8-42,8$ \\
\hline Valor de p & 0,003 & & \\
\hline \multicolumn{4}{|l|}{ Cor/Raça } \\
\hline Branca & 112 & 33,9 & $25,3-43,5$ \\
\hline Negra e mulatos & 103 & 21,4 & $13,9-30,5$ \\
\hline Valor de $\mathrm{p}$ & 0,040 & & \\
\hline \multicolumn{4}{|l|}{ Estado civil } \\
\hline Casado & 106 & 30,2 & $21,7-39,9$ \\
\hline Solteiro e outros & 111 & 26,1 & $18,2-35,3$ \\
\hline Valor de $\mathrm{p}$ & 0,505 & & \\
\hline \multicolumn{4}{|l|}{ Escolaridade } \\
\hline Até o Ensino Médio incompleto & 111 & 28,8 & $20,6-38,2$ \\
\hline Ensino Médio completo ou mais & 106 & 27,3 & $19,1-36,9$ \\
\hline Valor de $\mathrm{p}$ & 0,809 & & \\
\hline \multicolumn{4}{|l|}{ Classificação econômica * } \\
\hline Classes A e B & 87 & 32,2 & $22,1-43,1$ \\
\hline Classe C & 111 & 27,0 & $19,0-36,3$ \\
\hline Classes D e E & 19 & 15,8 & $3,4-39,6$ \\
\hline Valor de $\mathrm{p}$ & 0,331 & & \\
\hline \multicolumn{4}{|l|}{ Situação de trabalho } \\
\hline Com trabalho & 176 & 29,5 & $22,9-36,9$ \\
\hline Sem trabalho & 41 & 22,0 & $10,6-37,6$ \\
\hline Valor de $\mathrm{p}$ & 0,329 & & \\
\hline \multicolumn{4}{|l|}{ Local de nascimento } \\
\hline Juiz de Fora & 149 & 30,9 & $23,6-39,0$ \\
\hline Outros & 68 & 22,1 & $12,9-33,8$ \\
\hline Valor de $p$ & 0,180 & & \\
\hline
\end{tabular}

* De acordo com o critério da Associação Brasileira de Empresas de Pesquisa 30.

ciação ao sedentarismo/atividade física irregular foram a idade e a cor/raça dos entrevistados. Quanto maior a faixa etária, maior a proporção de sedentários e sem atividade física regular. Os brancos apresentaram menor índice de atividade física do que os negros/outros.

Em relação ao uso do tabaco (Tabela 4), quanto menor a escolaridade do entrevistado, maior a prevalência do tabagismo. Essa prevalência foi menor entre aqueles que referiram estar trabalhando do que entre os que não referiram atividade laboral. Quanto às características demográficas, observa-se que o consumo atual de cigarros aumentou conforme o aumento da faixa etária, e que a situação de fumante foi maior entre os que não eram naturais da cidade de Juiz de Fora.

Não houve diferenças estatisticamente significativas entre as prevalências de hipertensão ar- terial nos diferentes extratos das variáveis exploratórias (Tabela 5). Já a pressão arterial limítrofe aferida foi mais prevalente nos maiores de trinta anos, entre negros e mulatos, entre os casados, entre os que referiam estar empregados no momento da pesquisa e entre os que não haviam nascido em Juiz de Fora.

\section{Discussão}

No Brasil, ainda persiste uma carência de estudos de prevalência de doenças cardiovasculares e dos seus fatores de risco modificáveis no contexto populacional. Nesse universo restrito, nota-se que a maioria das pesquisas já realizadas utilizou populações da Região Sudeste 9 . Entretanto, Minas Gerais ainda tem participação 
Prevalência do uso atual do tabaco em homens entre 20 e 49, anos adscritos à Estratégia Saúde da Família da unidade básica de saúde do Alto Grajaú, Juiz de Fora, Minas Gerais, Brasil, em 2006.

\begin{tabular}{|c|c|c|c|}
\hline \multirow[t]{2}{*}{ Características sócio-demográficas } & \multirow[t]{2}{*}{$\mathrm{n}$} & \multicolumn{2}{|c|}{ Fumantes } \\
\hline & & $\%$ & IC95\% \\
\hline \multicolumn{4}{|l|}{ Faixa etária (anos) } \\
\hline$<30$ & 72 & 18,1 & $10,0-28,9$ \\
\hline$\geq 30$ & 145 & 29,0 & $21,7-37,1$ \\
\hline Valor de $\mathrm{p}$ & 0,082 & & \\
\hline \multicolumn{4}{|l|}{ Cor/Raça } \\
\hline Branca & 112 & 21,4 & $14,2-30,2$ \\
\hline Negra e mulatos & 103 & 29,1 & $20,6-38,9$ \\
\hline Valor de $p$ & 0,194 & & \\
\hline \multicolumn{4}{|l|}{ Estado civil } \\
\hline Casado & 106 & 21,7 & $14,3-30,8$ \\
\hline Solteiro e outros & 111 & 28,8 & $20,6-32,2$ \\
\hline Valor de $\mathrm{p}$ & 0,227 & & \\
\hline \multicolumn{4}{|l|}{ Escolaridade } \\
\hline Até o Ensino Médio incompleto & 111 & 35,1 & $26,3-44,8$ \\
\hline Ensino Médio completo ou mais & 106 & 15,1 & $8,9-23,4$ \\
\hline Valor de $\mathrm{p}$ & 0,001 & & \\
\hline \multicolumn{4}{|l|}{ Classificação econômica * } \\
\hline Classes A e B & 87 & 18,4 & $10,9-28,1$ \\
\hline Classe C & 111 & 30,6 & $22,2-40,1$ \\
\hline Classes D e E & 19 & 26,3 & $9,1-51,2$ \\
\hline Valor de p & 0,144 & & \\
\hline \multicolumn{4}{|l|}{ Situação de trabalho } \\
\hline Com trabalho & 176 & 21,0 & $15,3-27,8$ \\
\hline Sem trabalho & 41 & 43,9 & $28,5-60,3$ \\
\hline Valor de $\mathrm{p}$ & 0,002 & & \\
\hline \multicolumn{4}{|l|}{ Local de nascimento } \\
\hline Juiz de Fora & 149 & 21,5 & $15,2-28,9$ \\
\hline Outros & 68 & 33,8 & $22,8-46,3$ \\
\hline Valor de $p$ & 0,052 & & \\
\hline
\end{tabular}

* De acordo com o critério da Associação Brasileira de Empresas de Pesquisa 30.

discreta nesse cenário de estudos, que abordam principalmente populações do Rio de Janeiro e São Paulo. A cidade de Juiz de Fora, embora apresentando as doenças do aparelho circulatório como primeira causa de morte a partir dos vinte anos de idade (Departamento de Informática do SUS. Indicadores e dados básicos - Brasil-2005. IDB-2005. http:// tabnet.datasus. gov.br/cgi/idb2005/matriz.htm, acessado em 02/Dez/2006), também não foge a essa regra, sendo praticamente inexistentes inquéritos de saúde sobre o tema. Visando a compreender as semelhanças e especificidades desse subgrupo populacional com relação às demais populações, etapa fundamental para a elaboração de propostas efetivas de enfrentamento do problema, vale a pena comparar os principais achados do estudo com pesquisas anteriores realizadas em outras partes do mundo e do Brasil, vis-a-vis as características sócio-demográficas dos indivíduos que compõem a amostra do estudo.

De forma geral, o perfil demográfico da amostra é semelhante ao da população de Juiz de Fora e da Região Sudeste do país 31,33. A maior prevalência de brancos do estudo também foi encontrada na PNAD em 2002, quando os brancos representavam 62,1\% dos homens da Região Sudeste do Brasil 31. Na cidade de Juiz de Fora, em 2000, os homens também predominavam como chefes das famílias $(69,07 \%) 33$. Próximo da média brasileira (60\%) 31, em torno de $70 \%$ dos homens entrevistados referiram ser naturais da própria cidade. Embora com proporção diferente da relatada pela Associação Brasileira de Empresas de Pesquisas (ABEP) 30 para todo o Brasil no ano 2000, que indicava apenas $36 \%$ das famílias 
Prevalência de hipertensão arterial e pressão arterial limítrofe em homens entre 20 e 49 anos, adscritos à Estratégia Saúde da Família da unidade básica de saúde do Alto Grajaú, Juiz de Fora, Minas Gerais, Brasil, em 2006.

\begin{tabular}{|c|c|c|c|c|c|}
\hline \multirow[t]{2}{*}{ Características sócio-demográficas } & \multirow[t]{2}{*}{$\mathbf{n}$} & \multicolumn{2}{|c|}{ Hipertensão arterial aferida ou referida * } & \multicolumn{2}{|c|}{ Pressão arterial limítrofe aferida ** } \\
\hline & & $\%$ & IC95\% & $\%$ & IC95\% \\
\hline \multicolumn{6}{|l|}{ Faixa etária (anos) } \\
\hline$<30$ & 72 & 29,2 & $19,0-41,1$ & 9,7 & $4,0-19,0$ \\
\hline$\geq 30$ & 145 & 21,4 & $15,0-29,0$ & 24,1 & $17,4-31,9$ \\
\hline Valor de $p$ & & \multicolumn{2}{|c|}{0,206} & \multicolumn{2}{|c|}{0,010} \\
\hline \multicolumn{6}{|l|}{ Cor/Raça } \\
\hline Branca & 112 & 25,0 & $17,3-34,1$ & 14,4 & $8,4-22,2$ \\
\hline Negra e mulatos & 103 & 22,3 & $14,7-31,6$ & 24,3 & $16,4-33,7$ \\
\hline Valor de $\mathrm{p}$ & & \multicolumn{2}{|c|}{0,252} & \multicolumn{2}{|c|}{0,06} \\
\hline \multicolumn{6}{|l|}{ Estado civil } \\
\hline Casado & 106 & 27,4 & $19,1-36,9$ & 25,5 & $17,5-34,9$ \\
\hline Solteiro e outros & 111 & 20,7 & $13,6-29,5$ & 13,5 & $7,8-21,3$ \\
\hline Valor de $\mathrm{p}$ & & \multicolumn{2}{|c|}{0,252} & \multicolumn{2}{|c|}{0,026} \\
\hline \multicolumn{6}{|l|}{ Escolaridade } \\
\hline Até o Ensino Médio incompleto & 111 & 22,5 & $15,1-31,4$ & 20,7 & $13,6-29,5$ \\
\hline Ensino Médio completo ou mais & 106 & 25,0 & $17,5-34,9$ & 17,9 & $11,2-26,6$ \\
\hline Valor de $\mathrm{p}$ & & \multicolumn{2}{|c|}{0,611} & \multicolumn{2}{|c|}{0,600} \\
\hline \multicolumn{6}{|l|}{ Classificação econômica *** } \\
\hline Classes A e B & 87 & 26,4 & $17,6-37,0$ & 14,9 & $8,2-24,2$ \\
\hline Classe C & 111 & 20,7 & $13,6-29,5$ & 24,3 & $16,7-33,4$ \\
\hline Classes D e E & 19 & 31,6 & $12,6-56,6$ & 10,5 & $1,3-33,1$ \\
\hline Valor de $p$ & & \multicolumn{2}{|c|}{0,464} & \multicolumn{2}{|c|}{0,150} \\
\hline \multicolumn{6}{|l|}{ Situação de trabalho } \\
\hline Com trabalho & 176 & 23,9 & $17,8-30,9$ & 22,7 & $16,8-29,6$ \\
\hline Sem trabalho & 41 & 24,4 & $12,4-40,3$ & 4,9 & $0,6-16,5$ \\
\hline Valor de $\mathrm{p}$ & & \multicolumn{2}{|c|}{0,943} & \multicolumn{2}{|c|}{0,009} \\
\hline \multicolumn{6}{|l|}{ Local de nascimento } \\
\hline Juiz de Fora & 149 & 26,2 & $19,3-34,0$ & 15,4 & $10,0-22,3$ \\
\hline Outros & 68 & 19,1 & $10,6-30,5$ & 27,9 & $17,7-40,1$ \\
\hline Valor de $p$ & & \multicolumn{2}{|c|}{0,259} & \multicolumn{2}{|c|}{0,031} \\
\hline
\end{tabular}

* Foram considerados neste grupo aqueles que referiram hipertensão arterial e estar sob tratamento, e aqueles cujos valores da aferição foram iguais ou maiores do que $140 \times 90 \mathrm{mmHg}$;

** Foram considerados limítrofes aqueles que apresentavam pressão arterial no momento da entrevista entre 130-139mmHg x 85-90mmHg, retirando-se os que faziam uso de medicação para hipertensão;

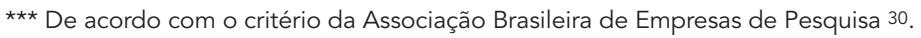

como da classe $\mathrm{C}$, ambos os estudos apontaram esta classe econômica como a dominante. Fugindo da situação do restante da região, $81,1 \%$ dos entrevistados relataram alguma atividade remunerada, situação mais favorável do que a indicada pelo Instituto Brasileiro de Geografia e Estatística (IBGE) para a população masculina da Região Sudeste em 2002 (65,7\%) 31.

Chama a atenção a alta prevalência de indivíduos com pelo menos um dos fatores de risco estudados, considerando que a amostra do estudo congregou adultos jovens, reforçando a necessidade de implementação de ações que visem à promoção da saúde e à prevenção de do- enças cardiovasculares já nas faixas etárias mais jovens da população 7,8,16. Da mesma forma, a simultaneidade de dois ou mais dos fatores de risco em um mesmo indivíduo esteve presente em quase $50 \%$ dos homens entrevistados. A alta concomitância também foi encontrada em estudos anteriores no país 12,18 e em outras partes do mundo 34,35 , indicando que as estratégias de promoção da saúde e controle das doenças cardiovasculares devem abordar o conjunto de fatores de risco, não se restringindo a um ou outro de forma isolada 1 .

A prevalência elevada de sobrepeso/obesidade não é particularidade da população do estudo. 
Dados provenientes de 48 diferentes países entre 1983 e 1986 mostraram que entre $50 \%$ e $70 \%$ da população de adultos com idades entre 35 e 64 anos estavam acima do peso ou eram obesos 36 . Nos Estados Unidos, houve aumento contínuo da prevalência do excesso de peso entre os anos de 1999 (27,5\%) e 2004 (31,1\%) 37. No Brasil, em 1989 , a obesidade (IMC $\geq 30$ ) entre homens acima de 18 anos era de $4,8 \%$ e o sobrepeso de $22,5 \% 17$. Estudos nacionais mais recentes reafirmam altas magnitudes 38,39,40. Acompanhando a tendência mundial, o presente estudo também evidenciou maior freqüência de sobrepeso e/ou obesidade entre os homens casados e de maior faixa etária.

Ao se analisar a prevalência de sedentarismo/atividade física irregular do estudo (cerca de $30 \%$ ), observa-se que esta é menor do que a prevista pela OMS para a população mundial (cerca de 50\%) 7, mas se assemelha à situação americana. Em 2002, resultados do BRFSS, que considerou 35 estados e o distrito de Columbia, indicavam que $22,3 \%$ dos homens não realizavam tais atividades, sendo que entre os mais velhos e os menos favorecidos economicamente a prática era menos freqüente 41 . A comparação dos achados do estudo com os da pesquisa americana deve ser feita com reservas, pois enquanto a realizada nos Estados Unidos se restringiu às atividades físicas de lazer, o presente estudo também considerou as atividades ocupacionais, de locomoção e relativas ao trabalho doméstico. A restrição à faixa etária de 20 a 49 anos também pode ser responsável pela menor prevalência encontrada em Juiz de Fora vis-a-vis aos outros estudos, pois a maioria das pesquisas indica que a inatividade física aumenta com a idade 7,19,42,43.

Os resultados das pesquisas brasileiras sobre o tema são heterogêneos. Estudo que utilizou o mesmo instrumento de aferição estimou prevalências de "insuficientemente ativos" (sedentários + irregularmente ativos) entre pessoas de 15 a 69 anos de ambos os sexos, que variaram entre 54,5\%, encontrada na cidade de João Pessoa, Paraíba, e 28,2\% na cidade de Belém, Pará 8 . A prevalência de $28 \%$ de sedentarismo/irregularmente ativos do presente estudo se compara com a encontrada em Belém, por exemplo, mas é inferior à encontrada em São Paulo, entre homens de 14 a 77 anos, no ano de 2002 (45,6\%) 15. Quando a análise se restringe apenas aos considerados "sedentários", os dados deste estudo indicam uma prevalência em torno de $12 \%$ (não mostrados), semelhante à estimada para São Paulo (10,4\%) e inferior à de Pelotas, Estado do Rio Grande do Sul $(24,1 \%)$, de acordo com pesquisa realizada em 200243.

Quanto ao perfil nos diferentes subgrupos populacionais, as pesquisas indicam que a ina- tividade física de lazer é mais prevalente entre os homens mais velhos e de maior escolaridade 42,44 . Quando se considera qualquer tipo de atividade física, principalmente aquelas relacionadas aos meios de transporte freqüentemente utilizados e ao tipo de trabalho, a inatividade física passa a ser mais freqüente entre os de classe social mais favorecida. É possível que a situação de Juiz de Fora seja conseqüência do perfil da amostra estudada, que congrega apenas homens jovens e que têm suas atividades físicas relacionadas ao trabalho, à locomoção ou às atividades do lar.

Apesar de alta, a prevalência de $25,3 \%$ de fumantes de cigarros foi inferior aos $47 \%$ apresentados pela OMS para a população mundial de homens (independente da idade) ${ }^{6}$. Quando comparada com os dados do National Health Interview Survey (NHIS) de 2005, relativos aos homens maiores de 18 anos residentes nos Estados Unidos (23,9\%) 45, percebe-se semelhança entre os achados. Assim como o que foi encontrado em Juiz de Fora, o perfil de usuários do estudo americano também indicou maior consumo entre os negros, naqueles com escolaridade mais baixa e com situação sócio-econômica menos favorecida.

No contexto brasileiro, a prevalência de tabagismo aproximou-se da apresentada pelo inquérito nacional realizado entre 2002 e 2003, para homens da capital mineira, 26,1\% ${ }^{8}$. Magnitude similar também foi estimada em inquéritos domiciliar (26,6\%) e por telefone (23,8\%), realizados na cidade de São Paulo 11,21. Quanto ao perfil do usuário, os achados da pesquisa também concordam com os apontados em pesquisas nacionais anteriores, indicando que a prevalência é maior entre os maiores de trinta anos, de nível sócio-econômico menos favorecido e sem vínculo empregatício 11,18,21. Em relação a essa última característica, a menor prevalência do tabagismo entre os trabalhadores pode ser devido aos programas empresariais de controle do tabaco, como foi atribuído no estudo de uma estatal nacional, em que o estímulo para o abandono do cigarro em curso na empresa foi apontado como possível determinante para a baixa prevalência do tabagismo $(12,4 \%) 13$.

A prevalência de hipertensão arterial da população estudada (24\%) foi semelhante à encontrada em estudo realizado com homens nos Estados Unidos, entre os anos de 2001 e 200446. No Brasil, a prevalência da hipertensão foi revista por Lessa 47 , e seus respectivos fatores de risco por Bloch et al. 9. De acordo com os autores, mesmo levando em consideração as diferenças metodológicas que dificultam uma abordagem conjunta dos achados, a prevalência do pro- 
blema nas diferentes regiões do país pode ser considerada alta. De acordo com a literatura, as prevalências variam entre $15 \%$ e $36,4 \%$ e dependem da população estudada e dos métodos utilizados na definição e aferição da pressão arterial $1,13,16,18,21,48,49$.

Apesar do seu potencial papel como fator de risco para a morbimortalidade por doenças cardiovasculares 5,50, as estimativas relativas à prevalência da pressão arterial normal alta na população, classificada como limítrofe pela Sociedade Brasileira de Cardiologia 28 e como pré-hipertensão pelo Seventh Report of The Joint National Committee on Prevention, Detection, Evaluation, and Treatment of High Blood Pressure (JNC 7) 3, não podem ser comparadas às obtidas em outros contextos nacionais, pois este é o primeiro estudo brasileiro que estima a prevalência do problema. Ao se considerar sua distribuição de acordo com as variáveis sócio-demográficas avaliadas, percebem-se prevalências bastante diferentes entre os vários subgrupos populacionais. Como apresentado na Tabela 5, esse nível de pressão arterial esteve mais presente entre os indivíduos maiores de trinta anos, entre negros ou mulatos, entre casados, entre os que relatavam ter emprego no momento da pesquisa e entre os que não haviam nascido em Juiz de Fora. Ao se comparar esse perfil ao dos indivíduos com hipertensão arterial já instalada, nota-se que uma relação inversa entre as prevalências. De modo geral, os estratos que apresentam maior prevalência de pressão arterial limítrofe são aqueles de menor prevalência de hipertensão arterial, o que pode sugerir que a pressão arterial limítrofe seja o estágio inicial da hipertensão arterial.

\section{Considerações finais}

A interpretação dos resultados da pesquisa deve ser feita à luz de algumas limitações metodológicas. A primeira delas diz respeito à aferição da pressão arterial sistêmica realizada com aparelho aneróide e sem a relação ótima entre o perímetro do braço e a largura do manguito. É possível que a estratégia simplificada de aferição de pressão arterial possa ter alterado a prevalência de hipertensão arterial, uma vez que a leitura do esfigmomanômetro é passível de falha humana, e a inadequação do tamanho do manguito pode levar a valores mal estimados da pressão arterial sistêmica. Mesmo considerando que o uso de aparelho digital confiável e a mensuração do perímetro do braço para adequação do manguito melhorariam a qualidade dos dados, entende-se que essa limitação não inviabiliza a utilização dos achados, desde que sejam considerados como uma apreciação inicial e exploratória do problema.

Outra questão importante é a constatação de que outros fatores de risco modificáveis, tais como o alcoolismo e os fatores metabólicos, deixaram de ser abordados no presente estudo. Entendendo-se que a simultaneidade de fatores de risco acentua o risco de ocorrência e morte por doenças cardiovasculares, é interessante que novos estudos se juntem ao realizado para que ações de prevenção e controle do problema, com efeitos mais abrangentes, possam ser propostas.

Finalmente, deve ser ressaltado o importante papel das equipes de saúde da família como facilitadoras da realização do inquérito e algumas das potencialidades da Estratégia Saúde da Família na vigilância dos fatores de risco para as doenças e agravos não-transmissíveis. $\mathrm{O}$ elo entre os entrevistadores e a comunidade só foi possível graças à participação ativa dos Agentes Comunitários de Saúde. A realização da pesquisa em parceria com equipes de saúde da família também permitiu uma ampla discussão entre pesquisadores e profissionais de saúde sobre as várias possibilidades de atuação das equipes frente à prevenção das doenças e agravos nãotransmissíveis e a vigilância concomitante de seus vários fatores de risco.

Dentre as várias possibilidades de atuação das equipes, constatou-se a importância da elaboração de diagnósticos locais amplos e atualizados que dêem subsídios ao planejamento de ações de promoção da saúde e prevenção das doenças cardiovasculares e demais doenças crônico-degenerativas. Nesse contexto destacase a importância da Estratégia Saúde da Família no monitoramento dos fatores de risco na população masculina. Considerando a baixa freqüência desse subgrupo populacional aos serviços de saúde, a utilização das visitas domiciliares e a integração dos Agentes Comunitários de Saúde às comunidades - pilares centrais da Estratégia Saúde da Família - passam a ter papel fundamental na detecção de situações de risco e implementação de propostas de ação especificamente voltadas para este subgrupo. Por fim, ressalta-se que a incorporação da Estratégia Saúde da Família aos sistemas de vigilância das doenças e agravos não-transmissíveis facilitaria o desenvolvimento de estudos nas diferentes regiões brasileiras, gerando as informações necessárias para alimentar um banco de dados abrangente e representativo dos diferentes cenários do país o que, certamente, possibilitaria a elaboração de estratégias de ação que considerem as diferentes nuances do problema. 


\section{Resumo}

O monitoramento dos fatores de risco para as doenças cardiovasculares vem sendo indicado em todo o mundo. Nesse sentido, o objetivo do estudo foi estimar a prevalência de tabagismo, sobrepeso/obesidade, atividade física insuficiente, hipertensão arterial e pressão arterial limítrofe entre homens adscritos à Estratégia Saúde da Família em área urbana de Juiz de Fora, Minas Gerais, Região Sudeste do Brasil. Foi realizado um inquérito domiciliar com 217 homens, entre 20 e 49 anos, selecionados por meio de amostragem sistemática baseada em listagem de moradores desta faixa etária. O sobrepeso/obesidade foi o fator de risco de maior freqüência (43,3\%; IC95\%: 36,6-50,2). Os sedentários e irregularmente ativos fisicamente totalizaram 28\% (IC95\%: 22,2-34,6). O consumo de cigarros era hábito de 25,3\% (IC95\%: 19,7-31,7) da população. Foram classificados como hipertensos $24 \%$ (IC95\%: 18,430,2) dos entrevistados e 19,4\% (IC95\%: 14,3-25,2) com pressão arterial limítrofe. A simultaneidade de dois ou mais fatores de risco foi encontrada em $45,2 \%$ da população. A magnitude das prevalências sugere que é preciso intensificar as estratégias de promoção de saúde voltadas para a população masculina.

Doenças Cardiovasculares; Saúde do Homem; Fatores de Risco

\section{Colaboradores}

E. B. B. D. Van Eyken foi responsável pelo trabalho de campo, análise dos dados e elaboração do artigo. C. L. Moraes foi orientadora e responsável pela pesquisa, análise dos dados e confecção do artigo.

\section{Referências}

1. Noncommunicable Diseases and Mental Health, World Health Organization. Integrated management of cardiovascular risk: eport of a WHO meeting, Geneva 9-12, July 2002. Geneva: World Health Organization; 2002.

2. Mackay J, Mensah GA. The atlas of heart disease and stroke. Geneva: World Health Organization; 2004.

3. Chobanian AV, Bakris GL, Black HR, Cushman WC, Green LA, Izzo Jr. JL, et al. Seventh Report of the Joint National Committee on Prevention, Detection, Evaluation and Treatment of High Blood Pressure. Hypertension 2003; 42:1206-52.

4. Lewington S, Clarke R, Qizilbash N, Peto R, Collins R; Prospective Studies Collaboration. Age-specific relevance of usual blood pressure to vascular mortality: a meta-analysis of individual data for one million adults in 61 prospective studies. Lancet 2002; 360:1903-13.

5. Vasan RS, Larson MG, Leip EP, Evans JC, O'Donnell CJ, Kannel WB, et al. Impact of high normal blood pressure on the risk of cardiovascular disease. $\mathrm{N}$ Engl J Med 2001; 345:1291-7.

6. World Health Organization. Building blocks for tobacco control. Geneva: World Health Organization; 2004.

7. World Health Organization. The World Health Report 2002: reducing the risks, promoting healthy life. http://www.who.int/whr/2002/en/ (acessado em 10/Jun/2005).

8. Instituto Nacional de Câncer. Inquérito domiciliar sobre comportamento de risco e morbidade referida de doenças e agravos não transmissíveis: Brasil, 15 capitais e Distrito Federal, 2002-2003. Rio de Janeiro: Instituto Nacional de Câncer; 2004. 
9. Bloch KV, Rodrigues CS, Fiszman R. Epidemiologia dos fatores de risco para hipertensão arterial: uma revisão crítica da literatura brasileira. Rev Bras Hipertens 2006; 13:134-43.

10. Feijão AMM, Gadelha FV, Bezerra AA, Oliveira AM, Silva MSS, Lima JWO. Prevalência de excesso de peso e hipertensão arterial em população urbana de baixa renda. Arq Bras Cardiol 2005; 84:29-33.

11. Marcopito LF, Rodrigues SSF, Pacheco MA, Shirassu MM, Goldfeder AJ, Moraes MA. Prevalência de alguns fatores de risco para doenças crônicas na cidade de São Paulo. Rev Saúde Pública 2005; 39:738-45.

12. Lessa I, Araújo MJ, Magalhães L, Almeida Filho N, Aquino E, Costa MCR. Simultaneidade de fatores de risco cardiovasculares modificáveis na população adulta de Salvador (BA), Brasil. Rev Panam Salud Pública 2004; 16:131-7.

13. Matos MFD, Silva NAS, Pimenta AJM, Cunha AJLA. Prevalência dos fatores de risco para a doença cardiovascular em funcionários do Centro de Pesquisas da Petrobrás. Arq Bras Cardiol 2004; 82:1-8.

14. Brandão AP, Brandão AA, Magalhães MEC, Pozzan R. Epidemiologia da hipertensão arterial. Rev Soc Cardiol Estado de São Paulo 2003; 13:7-19.

15. Matsudo SM, Matsudo VR, Araújo T, Andrade D, Andrade E, Oliveira L, et al. Nível de atividade física da população do Estado de São Paulo: análise de acordo com o gênero, idade, nível socioeconômico, distribuição geográfica e de conhecimento. Rev Bras Ciênc Mov 2002; 10:41-50.

16. Klein $\mathrm{CH}$, Silva NAS, Nogueira AR, Bloch KV, Campos LHS. Hipertensão arterial na Ilha do Governador, Rio de Janeiro, Brasil. I. Metodologia. Cad Saúde Pública 1995; 11:187-201.

17. Sichieri R, Coitinho DC, Leão MM, Recine E, Everhart JE. High temporal, geografic, and income variation in body mass index among adults in Brazil. Am J Public Health 1994; 84:793-7.

18. Ducan BB, Schimidt MI, Polanczyk CA, Homrich CS, Rosa RS, Achutti AC. Fatores de risco para doenças não transmissíveis em área metropolitana na região sul do Brasil: prevalência e simultaneidade. Rev Saúde Pública 1993; 27:43-8.

19. Centers for Disease Control and Prevention. BRFSS: turning information into health. http:// www.cdc.gov/brfss/ (acessado em 22/Fev/2005).

20. World Health Organization. Mega country health promotion network: behavioral risk factor surveillance guide. http://whqlibdoc.who.int/hq/2002/ a78626.pdf (acessado em 10/Ago/2005).

21. Monteiro CA, Moura EC, Jaime PC, Lucca A, Florindo AA, Figueiredo IRC, et al. Monitoramento de fatores de risco para doenças crônicas por entrevista telefônica. Rev Saúde Pública 2005; 39:47-57.

22. Moreira LB, Fuchs FD, Soares RS, Bredemir M, Cardozo S. Prevalência de tabagismo e fatores associados em área metropolitana da região sul do Brasil. Rev Saúde Pública 1995; 29:46-51.

23. Lessa I. Doenças crônicas não-transmissíveis no Brasil: um desafio para a complexa tarefa de vigilância. Ciênc Saúde Coletiva 2004; 9:931-43.

24. Ministério da Saúde. A vigilância, o controle e a prevenção das doenças crônicas não transmissíveis: DCNT no contexto do Sistema Único de Saúde brasileiro. Brasília: Organização Pan-Americana da Saúde; 2005.
25. Ministério da Saúde. O Programa Saúde da Família e a atenção básica no Brasil. Brasília: Ministério da Saúde; 2002.

26. Laurenti R, Mello Jorge MHP, Gotlieb SLD. Perfil epidemiológico da morbimortalidade masculina. Ciênc Saúde Coletiva 2005; 10:35-46.

27. Schraiber LB, Gomes R, Couto MT. Homens e saúde na pauta da saúde coletiva. Ciênc Saúde Coletiva $2005 ; 10: 7-17$.

28. Gomes MAM, Nobre F, Amodeo C, Kohlmann Jr. O, Praxedes JN, Machado CA, et al. IV diretrizes brasileiras de hipertensão arterial. Arq Bras Cardiol 2004; 82 Suppl 4:7-14.

29. Fonseca MJM, Faerstein E, Chór D, Lopes CS. Validade de peso e estatura informados e índice de massa corporal: Estudo Pró-Saúde. Rev Saúde Pública 2004; 38:392-8.

30. Associação Brasileira de Empresas de Pesquisa. Critério de classificação econômica Brasil. São Paulo: Associação Brasileira de Empresas de Pesquisa; 2003.

31. Instituto Brasileiro de Geografia e Estatística. Pesquisa Nacional por Amostra de Domicílio: síntese de indicadores - PNAD 2002. Rio de Janeiro: Instituto Brasileiro de Geografia e Estatística; 2003.

32. Pardini R, Matsudo S, Araújo T, Matsudo V, Andrade E, Braggion G, et al. Validação do questionário internacional de nível de atividade física (IPAQ - versão 6): estudo piloto em adultos jovens brasileiros. Rev Bras Ciênc Mov 2001; 9:45-51.

33. Centro de Pesquisas Sociais, Universidade Federal de Juiz de Fora. Anuário estatístico de Juiz de Fora: demografia. Juiz de Fora: Universidade Federal de Juiz de Fora; 2006.

34. Pleis JR, Lethbridge-Cejku M. Summary health statistics for U.S. adults: National Health Interview Survey, 2005. Vital Health Stat 10 2006; (232):1-153.

35. Hahn RA, Heath GW, Chang MH. Cardiovascular disease risk factors and preventive practices among adults - United States, 1994: a behavioral risk factor atlas. Behavioral Risk Factor Surveillance System State Coordinators. MMWR CDC Surveill Summ 1998; 47:35-69.

36. World Health Organization. Obesity: preventing and managing the global epidemic. Report of a WHO Consultation. Geneva: World Health Organization; 2000. (WHO Technical Report Series, 894).

37. Ogden CL, Carrol MD, Curtin LR, McDowell MA, Tabak CJ, Flegal KM. Prevalence of overweight and obesity in the United States, 1999-2004. JAMA 2006; 295:1549-55.

38. Instituto Brasileiro de Geografia e Estatística. Pesquisa de orçamentos familiares - POF 2002-2003: análise da disponibilidade domiciliar de alimentos e do estado nutricional no Brasil. http://www.ibge. gov.br/home/estatistica/populacao/condicaode vida/pof/2002analise/default.shtm (acessado em 15/Dez/ 2006)

39. Siqueira KS, Appolinario JC, Sichieri R. Overweight, obesity, and binge eating in a non-clinical sample of five Brazilian cities. Obes Res 2004; 12:1921-4.

40. Velásquez-Meléndez G, Pimenta AD, Kac G. Epidemiologia do sobrepeso e da obesidade e seus fatores determinantes em Belo Horizonte (MG), Brasil: estudo transversal de base populacional. Rev Panam Salud Pública 2004; 16:308-14. 
41. Centers for Disease Control and Prevention. Prevalence of no-leisure time physical activity: 35 States and the District of Columbia, 1988-2002. MMWR Morb Mortal Wkly Rep 2004; 53:82-6.

42. Monteiro CA, Conde WL, Matsudo SM, Matsudo VR, Bonseñor IM, Lotufo PA. A descriptive epidemiology of leisure-time physical activity in Brazil, 1996-1997. Rev Panam Salud Pública 2003; 14: 246-54.

43. Hallal PC, Matsudo SM, Matsudo VKR, Araújo TL, Andrade DR, Bertoldi AD. Physical activity in adults from two Brazilian areas: similarities and differences. Cad Saúde Pública 2005; 21:573-80.

44. Gomes VB, Siqueira KS, Sichieri R. Atividade física em uma amostra probabilística da população do Município do Rio de Janeiro. Cad Saúde Pública 2001; 17:969-76.

45. Centers for Disease Control and Prevention. Racial/ethnic and socioeconomic disparities in multiple risk factors for heart disease and stroke: United States, 2003. MMWR Morb Mortal Wkly Rep 2005; 54:113-7.
46. U.S. Department of Health and Human Services, Centers for Disease Control and Prevention, National Center for Health Statistics. Health, United States, 2006: with chartbook on trends in health of Americans. Hyattsville: National Center for Health Statistics; 2006. (DHHS Publication, 2006-1232).

47. Lessa I. Epidemiologia da pressão arterial sistêmica e da insuficiência cardíaca no Brasil. Rev Bras Hipertens 2001; 8:383-92.

48. Barreto SM, Passos VMA, Cardoso ARA, LimaCosta MF. Quantificando o risco da doença coronariana na comunidade. Projeto Bambuí. Arq Bras Cardiol 2003; 81:549-55.

49. Trindade IS, Heineck G, Machado JR, Ayzemberg H, Formighieri M, Cretani M, et al. Prevalência de hipertensão arterial sistêmica na população urbana de Passo Fundo (RS). Arq Bras Cardiol 1998; 71:127-30.

50. Wilson PWF, D’Agostinho RB, Levy D, Belanger AM, Silbershatz H, Kannel WB. Prediction of coronary heart disease using risk factors categories. Circulation 1998; 97:1837-47.

Recebido em 17/Mai/2007

Versão final reapresentada em 19/Mai/2008

Aprovado em 30/Mai/2008 